\title{
Resource Reduction Method for the LTE-Advanced Uplink ACK/NACK Signal and SR
}

\author{
Seigo NAKAO ${ }^{\dagger a)}$, Member, Kenji TAKAGI ${ }^{\dagger \dagger}$, Nonmember, Masaru FUKUOKA ${ }^{\dagger \dagger}$, Daichi IMAMURA ${ }^{\dagger}$, Members, , \\ Hidekazu MURATA ${ }^{\dagger \dagger}$, Senior Member, Koji YAMAMOTO ${ }^{\dagger \dagger}$, Member, and Susumu YOSHIDA ${ }^{\dagger \dagger \dagger}$, Fellow
}

\begin{abstract}
SUMMARY Advanced Evolved Universal Terrestrial Radio Access (Advanced E-UTRA), called LTE-Advanced, has been standardized in the 3rd Generation Partnership Project (3GPP) as a candidate for IMTAdvanced. LTE-Advanced supports spatial orthogonal-resource transmit diversity (SORTD) [1], [2] for ACK/NACK signals and scheduling requests (SRs), which are used to control downlink hybrid automatic repeat requests (HARQs) and manage uplink radio resources based on uplink data traffic, respectively. Both ACK/NACK signals and SRs are carried via a physical uplink control channel (PUCCH) [3], and a common PUCCH format is used for both ACK/NACK signals and SRs. If SORTD is used, the base station assigns mutually orthogonal resources to each antenna included in the user equipment (UE) for ACK/NACK signals and SRs; hence, the number of required resources increases with the number of transmitting antennas in the UE. In this paper, we study the resource reduction method for ACK/NACK signal and SR in case of SORTD using the concept of common resource. In addition, we investigate a phase rotation scheme for common resources to improve the SR detection performance.

key words: LTE-Advanced, space orthogonal resource transmit diversity, phase rotation, ACK/NACK signal, scheduling request
\end{abstract}

\section{Introduction}

In LTE-Advanced, two types of control information are carried via PUCCH. One is the ACK/NACK signal, which is modulated by binary phase-shift keying (BPSK) and controls downlink HARQs. The other control information is $\mathrm{SR}$, which indicates that the UE has uplink data to be transmitted so that the base station can assign uplink data resources as needed. On-off keying (OOK) is used to carry SRs, and the base station determines that the SR was sent from the UE if a certain power is detected at the PUCCH resource assigned to the SR (i.e., an SR resource) [4].

The base station assigns independent $\mathrm{PUCCH}$ resources for ACK/NACK signals and SRs to the UE. If the UE needs to transmit an ACK/NACK signal and an SR simultaneously, it uses the SR resource to carry ACK/NACK information. In other words, ACK/NACK signals modulated by BPSK are carried via the SR resource. The base station uses power detection to determine whether the SR

Manuscript received July 11, 2010.

Manuscript revised September 22, 2010.

${ }^{\dagger}$ The authors are with the Next-Generation Mobile Communications Development Center, Tokyo R\&D Center, Panasonic Corporation, Yokohama-shi, 224-8539 Japan.

${ }^{\dagger}$ The authors are with Panasonic Mobile Communications R\&D Lab. Co., Ltd., Kanazawa-shi, 920-0024 Japan.

${ }^{\dagger \dagger}$ The authors are with the Graduate School of Informatics, Kyoto University, Kyoto-shi, 606-8501 Japan.

a) E-mail: nakao.seigo@jp.panasonic.com

DOI: $10.1587 /$ transcom.E94.B.364 was sent, and it detects the ACK or NACK information using, e.g., coherent detection of the signal received via the resource.

SORTD is employed in LTE-Advanced to improve ACK/NACK signal and SR performance. The base station assigns mutually orthogonal $\mathrm{PUCCH}$ resources for all the antennas in the UE, increasing the PUCCH overhead. Moreover, the number of PUCCH resources required for ACK/NACK signals and SRs increases with the number of transmitting antennas in the UE; hence, the PUCCH overhead is considered a serious concern in the use of LTEAdvanced.

To reduce the PUCCH overhead, we propose the use of partially overlapping resources for SORTD of ACK/NACK signals and SRs. In addition, we investigate a novel phase rotation scheme for the resources commonly used for ACK/NACK signals and SRs that can improve SR detection performance. First, a brief overview of SORTD for ACK/NACK signals and SRs is given in Sect. 2. Details of the proposed scheme and its performance are provided in Sect. 3, and finally our conclusions are presented in Sect. 4.

\section{Overview}

This section provides an overview of the use of SORTD for ACK/NACK signals and SRs in LTE-Advanced.

\subsection{ACK/NACK Signals and SRs in LTE-Advanced}

If the UE receives downlink data, it generates ACK/NACK information depending on the result of the downlink data reception and modulates the ACK/NACK information using BPSK.

Code spreading by a cyclic shift (CS) sequence of length 12 is performed for modulated ACK/NACK signals and a reference signal (RS) used for coherent detection. We denote the CS sequence, which has a CS value of $m$, as $\tilde{F}_{m}\left(n_{f}\right)$ in the frequency domain, where $n_{f}$ is the subcarrier index, as in [5].

Then, a second code spreading is performed using orthogonal sequences, i.e., a Walsh sequence and discrete Fourier transform (DFT) sequence, on the signals spread by the CS sequences. As a result, the modulated ACK/NACK signal is code spread onto the first, second, sixth, and seventh symbols, and the RS is code spread onto the third, fourth, and fifth symbols in normal cyclic prefix (CP) for- 


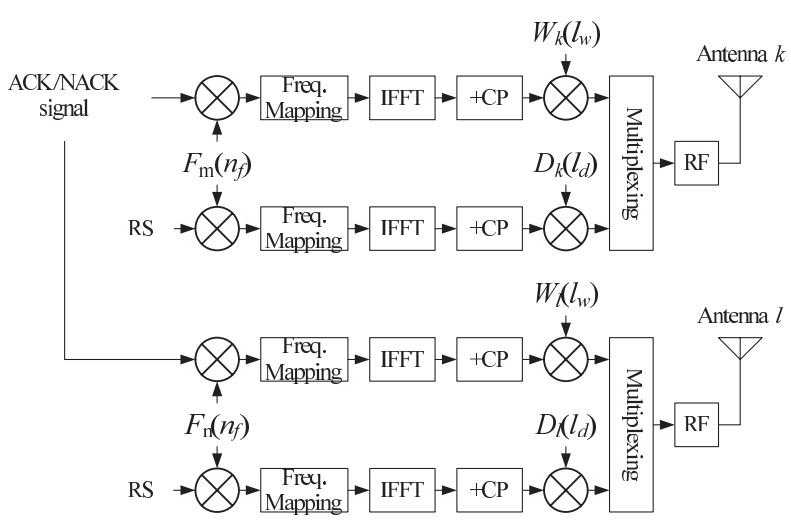

Fig. 1 Spatial orthogonal-resource transmit diversity (SORTD) for ACK/NACK signals and SRs.

mats [5]. Since ACK/NACK signals from different UE can be multiplexed using CDMA, the resource for sending the ACK/NACK signal is defined by a set consisting of a CS sequence and an orthogonal sequence.

The same PUCCH format is used for carrying the SR as for the ACK/NACK signal, and if the ACK/NACK signal and SR are generated simultaneously by the UE, the resource (i.e., a set consisting of a CS sequence and an orthogonal sequence) assigned to the SR is used to carry ACK/NACK information. The base station uses power detection of the SR resource to determine whether the SR was sent by the UE and derives the ACK/NACK information by coherent detection of the signal received via the resource.

\subsection{SORTD for ACK/NACK Signals and SRs in LTE- Advanced}

In LTE-Advanced, SORTD is used as a transmit diversity scheme for ACK/NACK signals and SRs [1], [2]. The base station assigns mutually orthogonal resources to all transmitting antennas in the UE, and the UE transmits the same signals using multiple antennas on different resources, as shown in Fig. 1. The base station combines the signals received via these resources, e.g., by using maximum ratio combining (MRC).

If the UE has two transmitting antennas, the base station assigns two PUCCH resources for ACK/NACK signals and another two for SRs; hence, the base station assigns a total of four PUCCH resources to one piece of UE.

\section{Proposed SORTD Scheme}

In this section, details of the proposed SORTD scheme, which can reduce PUCCH overhead and improve its performance, are presented.

\subsection{A Common Resource for ACK/NACK Signals and SRs when SORTD Is Used}

As discussed in the previous section, considering a simultaneous transmission of an ACK/NACK signal and SR,

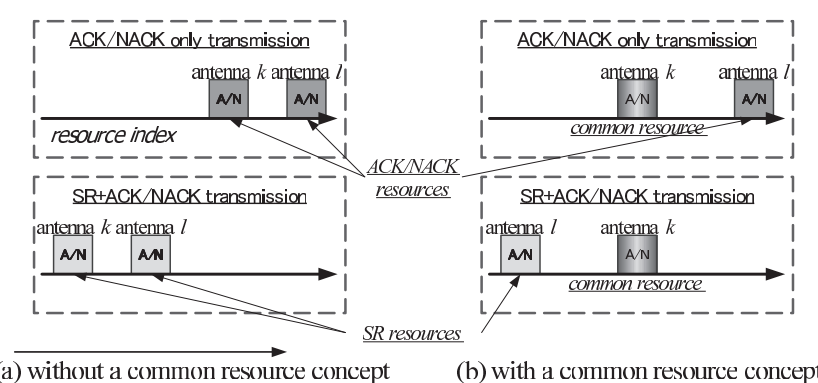

Fig. 2 Concept of the common resource.

the conventional SORTD scheme requires a total of four PUCCH resources, as shown in Fig. 2(a). Note that the horizontal axis of the figure indicates $\mathrm{PUCCH}$ resource indices used for ACK/NACK signals and SRs.

In order to reduce PUCCH overhead, one of the resources can be shared as shown in Fig. 2(b), since ACK/NACK signals and SRs are sent via resources that have the same physical format. In Fig. 2(b), the UE uses an ACK/NACK resource and a common resource when only an ACK/NACK signal is transmitted, and it uses an SR resource and a common resource when an ACK/NACK signal and SR are transmitted simultaneously.

If a common resource is not used [Fig. 2(a)], the power detected at the base station for a pair of SR resources when an ACK/NACK signal and SR are simultaneously transmitted $\left(P_{S R \rightarrow S R}\right)$ can be expressed as

$$
\begin{aligned}
P_{S R \rightarrow S R}= & 3\left|h_{k}^{S R} S_{R S}\right|^{2}+4\left|h_{k}^{S R} S_{A N}\right|^{2}+\sigma_{k, S R}^{2} \\
& +3\left|h_{l}^{S R} S_{R S}\right|^{2}+4\left|h_{l}^{S R} S_{A N}\right|^{2}+\sigma_{l, S R}^{2} \\
= & 7\left|h_{k}^{S R}\right|^{2}+\sigma_{k, S R}^{2}+7\left|h_{l}^{S R}\right|^{2}+\sigma_{l, S R}^{2},
\end{aligned}
$$

where $S_{R S}(=1)$ is the RS, $S_{A N}(= \pm 1)$ is the ACK/NACK signal, and $\sigma^{2}$ is the power of the noise. $h_{k}^{S R}$ is the effective propagation channel for the SR resource assigned to antenna $k$, and $h_{l}^{S R}$ is that for the SR resource assigned to antenna l. Similarly, the detected power of a pair of ACK/NACK resources when an ACK/NACK signal and SR are simultaneously transmitted $\left(P_{A N \rightarrow S R}\right)$ can be expressed as follows, since no signals are actually transmitted via ACK/NACK resources, as shown in Fig. 2(a).

$$
P_{A N \rightarrow S R}=\sigma_{k, A N}^{2}+\sigma_{l, A N}^{2}
$$

On the other hand, if a common resource is used, the detected power of the SR and common resources when ACK/NACK and SR are transmitted simultaneously $\left(P_{S R \rightarrow S R}^{c o m}\right)$ is

$$
P_{S R \rightarrow S R}^{c o m}=7\left|h_{k}^{c o m}\right|^{2}+\sigma_{k, \text { com }}^{2}+7\left|h_{l}^{S R}\right|^{2}+\sigma_{l, S R}^{2},
$$

where $h_{k}^{\text {com }}$ is the effective propagation channel of the common resource assigned to antenna $k$. The detected power of an ACK/NACK resource and a common resource when an ACK/NACK signal and SR are transmitted simultaneously $\left(P_{A N \rightarrow S R}^{c o m}\right)$ can be expressed as follows. 


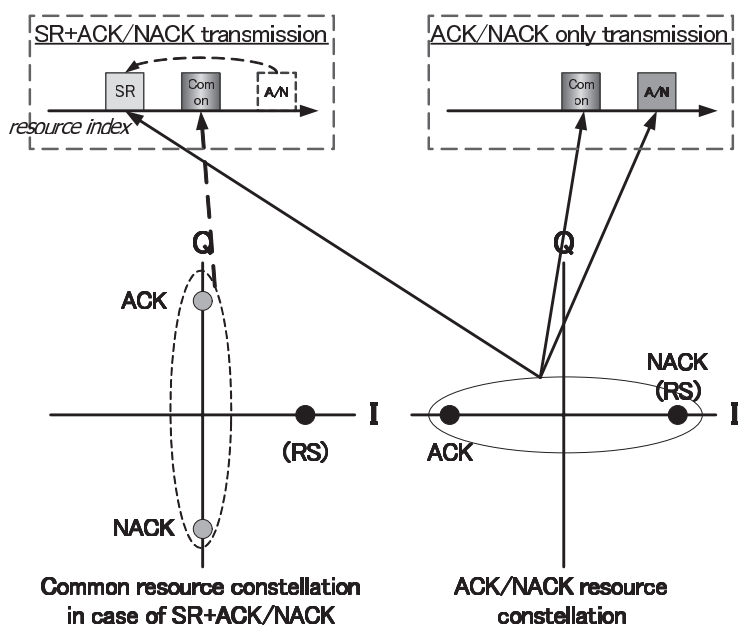

Fig. 3 Proposed phase rotation of the common resource.

$$
P_{A N \rightarrow S R}^{c o m}=7\left|h_{k}^{c o m}\right|^{2}+\sigma_{k, c o m}^{2}+\sigma_{l, A N}^{2}
$$

The base station determines whether an SR is transmitted by the power detected at the resource pairs. As Eqs. (1)-(4) show, the SR detection performance when using the common resource would be worse than that without the common resource. This is because only half of the transmitting power actually used by the UE can be used to distinguish the resource pair [see Eqs. (3) and (4)].

To mitigate performance degradation due to the use of a common resource, a novel phase rotation scheme for the common resource is proposed in the following subsection.

3.2 A Novel Phase Rotation Scheme for a Common Resource for SORTD

In brief, the proposed scheme rotates constellations of ACK/NACK signals by 90 degrees during simultaneous transmission of an ACK/NACK signal and an SR. As a result, the common resource uses different constellations when the UE sends only an ACK/NACK signal and when it sends an ACK/NACK signal and SR simultaneously, as shown in Fig. 3. At the base station, instead of using a power threshold to detect SRs, the Euclidean distance between the constellation point of the received signal and the nearest theoretical constellation point is used, as discussed below.

In the following discussions, $R_{c o m}^{e q}, R_{S R}^{e q}$, and $R_{A N}^{e q}$ represent the equalized signals received via the common resource, SR resource, and ACK/NACK resource, respectively. Assuming MRC is used, the signal detected via an $\mathrm{SR}$ resource and common resource when an ACK/NACK signal and an SR are simultaneously transmitted $\left(R_{S R \rightarrow S R}^{e q}\right)$ can be expressed by Eq. (5), since the ACK/NACK signal transmitted via the common resource is rotated 90 degrees in this case.

$$
\begin{aligned}
& R_{c o m}^{e q}=h_{k}^{c o m} j S_{A N}\left(h_{k}^{c o m}\right)^{-1} \\
& R_{S R}^{e q}=h_{l}^{S R} S_{A N}\left(h_{l}^{S R}\right)^{-1}
\end{aligned}
$$

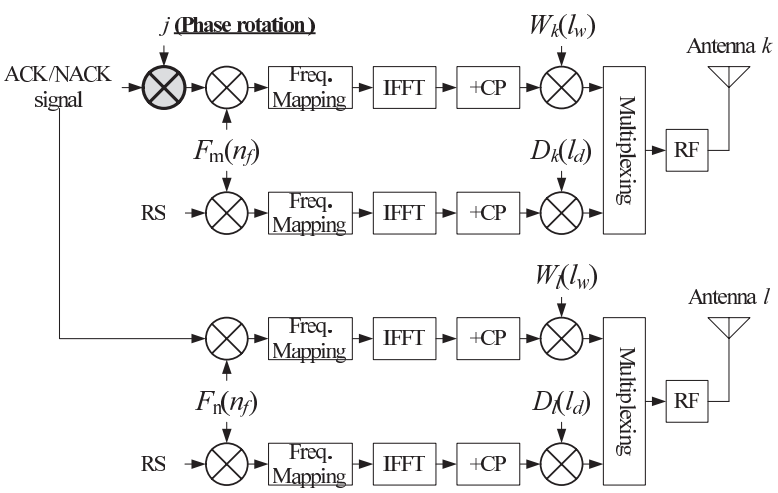

Fig. 4 Block diagram of the proposed transmitter with the phase rotation scheme.

$$
\begin{aligned}
R_{S R \rightarrow S R}^{e q} & =\frac{-j\left|h_{k}^{c o m}\right|^{2} R_{c o m}^{e q}+\left|h_{l}^{S R}\right|^{2} R_{S R}^{e q}}{\left|h_{k}^{c o m}\right|^{2}+\left|h_{l}^{S R}\right|^{2}}+\sigma_{S R \rightarrow S R} \\
& =S_{A N}+\sigma_{S R \rightarrow S R}
\end{aligned}
$$

$\sigma$ is the effect of noise.

On the other hand, no signal is actually transmitted via the ACK/NACK resource during simultaneous transmission of an ACK/NACK signal and SR. Hence, the signal detected via an ACK/NACK resource and common resource when an ACK/NACK signal and an SR are simultaneously transmitted $\left(R_{A N \rightarrow S R}^{e q}\right)$ can be expressed by Eq. (6), since the base station does not rotate $R_{c o m}^{e q}$ when it is combined with the signal detected at the ACK/NACK resource.

$$
R_{A N \rightarrow S R}^{e q}=j S_{A N}+\sigma_{A N \rightarrow S R}
$$

Comparing Eqs. (5) and (6) shows that the Euclidean distance between the constellation point of the received signal and the nearest theoretical constellation point (i.e., 1 or -1) for Eq. (6) is significantly larger than that for Eq. (5). Hence, SR detection using the Euclidean distance would be improved with the proposed phase rotation scheme on the common resource.

\subsection{Performance Evaluations}

This subsection presents simulation results for SR detection using the conventional and proposed SORTD schemes.

\subsubsection{Simulation Setup}

The transmitter is assumed to always send an ACK/NACK signal and an SR simultaneously in the simulation. At the transmitter, as shown in Fig. 4, the ACK/NACK signal is modulated by BPSK, and the signal transmitted from antenna $k$ via the common resource is multiplied by a complex value of $j$. Then, these modulated ACK/NACK signals and RSs used for coherent detection are code spread by the $\mathrm{CS}$ and orthogonal sequences. Note that different code resources (i.e., a set consisting of a CS sequence and an orthogonal sequence) are assigned to the common resource 


\begin{tabular}{c|c} 
Table 1 & Simulation conditions. \\
\hline Parameters & Values \\
\hline System bandwidth & $5[\mathrm{MHz}]$ \\
SC-FDMA symbol duration & $66.67[\mu \mathrm{s}]$ \\
& (except the CP part) \\
CP length & $4.7[\mu \mathrm{s}]$ \\
Antenna configurations & 2 Tx and 2 Rx \\
ACK/NACK Modulation & BPSK \\
Channel Model & 6-path Typical Urban [6] \\
Channel estimation & real \\
Mobility & $30[\mathrm{~km} / \mathrm{h}]$ \\
\hline
\end{tabular}

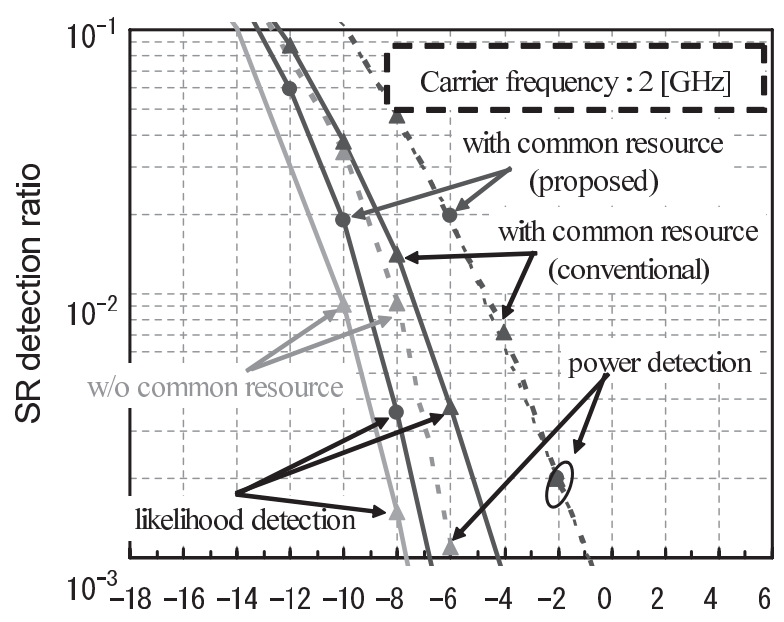

Average Signal to Noise Ratio $[\mathrm{dB}]$

Fig. 5 Performance evaluation results.

used by antenna $k$ and the SR resource used by antenna $l$.

The base station determines whether the SR is transmitted by calculating the received power [see Eqs. (1)-(4)] when power detection is used, and by calculating the Euclidean distance [see Eqs. (5), (6)] when likelihood detection is used. Other simulation conditions are summarized in Table 1.

\subsubsection{Simulation Results}

Figure 5 shows the SR detection performance with and without the proposed phase rotation scheme. In addition, as a reference, the SR detection performance when the UE does not use a common resource is also shown. The SR detection performance is defined as the percentage of correct SR detections by the base station, assuming that the UE always sends an ACK/NACK signal and an SR simultaneously. The $x$-axis of the graph indicates the average signal-to-noise ratio (SNR) of the ACK/NACK signal, and the $y$-axis indicates the SR detection performance.

If one resource is commonly used for the ACK/NACK signal and SR for SORTD, the SR detection performance is significantly degraded. This is because only half of the transmitting power actually used by the UE can be used to distinguish the resource pair, as discussed in the previous section. For instance, regardless of the detection scheme (i.e., power detection or likelihood detection), the required SNR for achieving the SR detection ratio of $10^{-2}$ becomes $3[\mathrm{~dB}]$ worse compared to the case when a common resource is not used, as shown in Fig. 5.

On the other hand, if the phase rotation is applied to the common resource, the Euclidean distance for the signal received via the SR and common resources can be further differentiated from that for the signal received via the ACK/NACK and common resources. As a result, the SR detection performance when using likelihood detection becomes comparable (i.e., less than $1[\mathrm{~dB}]$ degradation) to the case when the common resource is not used, although the SR detection performance when using power detection is not improved.

\section{Conclusion}

In this study, we first investigated the reduction of the PUCCH overhead caused by supporting SORTD in LTEAdvanced. Although the common resource concept can reduce the overhead by $25 \%$ when the UE has two transmitting antennas, we found that the SR detection performance is degraded because the receiving power that can be used for SR detection decreases.

To improve the SR detection performance, we proposed rotating the constellation of the common resource when it carries an SR, in conjunction with the use of likelihood detection at the base station. The results of a simulated performance evaluation confirmed that the SR detection performance is significantly improved by the proposed phase rotation scheme and is comparable to the case when no common resource is used.

\section{References}

[1] NTT DOCOMO, "UL transmit diversity schemes in LTE-Advanced," 3GPP TSG RAN WG1 Meeting 57, R1-092100, San Francisco, USA, May 2009.

[2] Qualcomm Europe, "PUCCH transmit diversity," 3GPP TSG RAN WG1 Meeting 57, R1-092065, San Francisco, USA, May 2009.

[3] A. Ghosh, R. Ratasuk, B. Classon, V. Nangia, R. Love, D. Schwent, and D. Wilson, "Uplink control channel design for 3GPP LTE," Proc. IEEE PIMRC'07, pp.1-5, May 2008.

[4] 3GPP, "TS 36.213 v8.8.0; Evolved universal terrestrial radio access (E-UTRA); Physical layer procedures," Sept. 2009.

[5] S. Nakao, T. Takata, M. Fukuoka, D. Imamura, K. Hiramatsu, and K. Miya, "A novel spreading code design for E-UTRA uplink control channel and its performance," IEICE Trans. Commun., vol.E92B, no.7, pp.2433-2441, July 2009.

[6] 3GPP, “TR 25.943 v7.0.0; Deployment aspects," June 2007. 